This is an electronic reprint of the original article. This reprint may differ from the original in pagination and typographic detail.

Author(s): Kähärä, Topi

Title: $\quad$ Numerical study of two-dimensional wet foam over a range of shear rates

Year: $\quad 2017$

Version:

Please cite the original version:

Kähärä, T. (2017). Numerical study of two-dimensional wet foam over a range of shear rates. Physical Review Fluids, 2(9), Article 093303.

https://doi.org/10.1103/PhysRevFluids.2.093303

All material supplied via JYX is protected by copyright and other intellectual property rights, and duplication or sale of all or part of any of the repository collections is not permitted, except that material may be duplicated by you for your research use or educational purposes in electronic or print form. You must obtain permission for any other use. Electronic or print copies may not be offered, whether for sale or otherwise to anyone who is not an authorised user. 


\title{
Numerical study of two-dimensional wet foam over a range of shear rates
}

\author{
T. Kähärä* \\ Department of Physics, University of Jyväskylä, PO Box 35, FI-40014 University of Jyväskylä, Finland
}

(Received 19 August 2016; published 8 September 2017)

\begin{abstract}
The shear rheology of two-dimensional foam is investigated over a range of shear rates with the numerical DySMaL model, which features dynamically deformable bubbles. It is found that at low shear rates, the rheological behavior of the system can be characterized by a yield stress power-law constitutive equation that is consistent with experimental findings and can be understood in terms of soft glassy rheology models. At low shear rates, the system rheology is also found to be subject to a scaling law involving the bubble size, the surface tension, and the viscosity of the carrier fluid. At high shear rates, the model produces a dynamic phase transition with a sudden change in the flow pattern, which is accompanied by a drop in the effective viscosity. This phase transition can be linked to rapid changes in the average bubble deformation and nematic order of the system. It is very likely that this phase transition is a result of the model dynamics and does not happen in actual foams.
\end{abstract}

DOI: 10.1103/PhysRevFluids.2.093303

\section{INTRODUCTION}

\section{A. Foam rheology}

Foams and emulsions have several interesting rheological properties, and there have been ongoing attempts to understand them [1,2]. However, because of the complicated nature of these materials, a mathematical framework for a comprehensive understanding of them has not been found, although different approaches can explain certain phenomena quite well. I have thus opted to approach the problem through simulation, which can fill in some pieces of the puzzle. Many simulations approach the problem of foam rheology from a dry foam perspective, usually in the quasistatic regime [3] or with out-of-equilibrium effects included [4]. Models for wet foam also exist [5-8]. In this work I use the DySMaL model, a Lagrangian model introduced in [9]. The model operates in the wet foam limit and has realistically deformable bubbles, a feature that becomes increasingly important at high shear rates or highly constrained flows.

\section{B. The DySMaL model}

In the DySMaL model, bubbles consist of individual subparticles (nodes) (see Fig. 1). Bubbles are considered to be suspended in a carrier fluid, which is not explicitly modeled but is partially taken into account in the interactions between bubbles. This approximation is reasonable in situations in which the fluid flow is heavily constrained by the bubbles. Geometry is constrained to two dimensions, and lengths in the third spatial dimension are fixed. Bubbles should therefore be imagined as cylinders in three dimensions. The circumference of a bubble is discretized into $N$ segments by $N$ subparticles, which are then individually propagated by the time integrator based on the forces applied to the corresponding segments of the surface. The interior of the bubble is considered to behave as a constant-temperature ideal gas, and thus it obeys the ideal gas law: $p_{\text {in }} V=w$, where $w$ is a bubble-specific constant. The bubble is held together by a surface-tension force $\boldsymbol{F}_{\text {surf }}$ between the ends of each segment, which in equilibrium was counterbalanced by the force $\boldsymbol{F}_{\text {pres }}$ arising from the pressure difference between the interior and exterior of the bubble. The surface-tension force is

*Author to whom all correspondence should be addressed: topi.kahara@jyu.fi 


\section{T. KÄHÄRÄ}

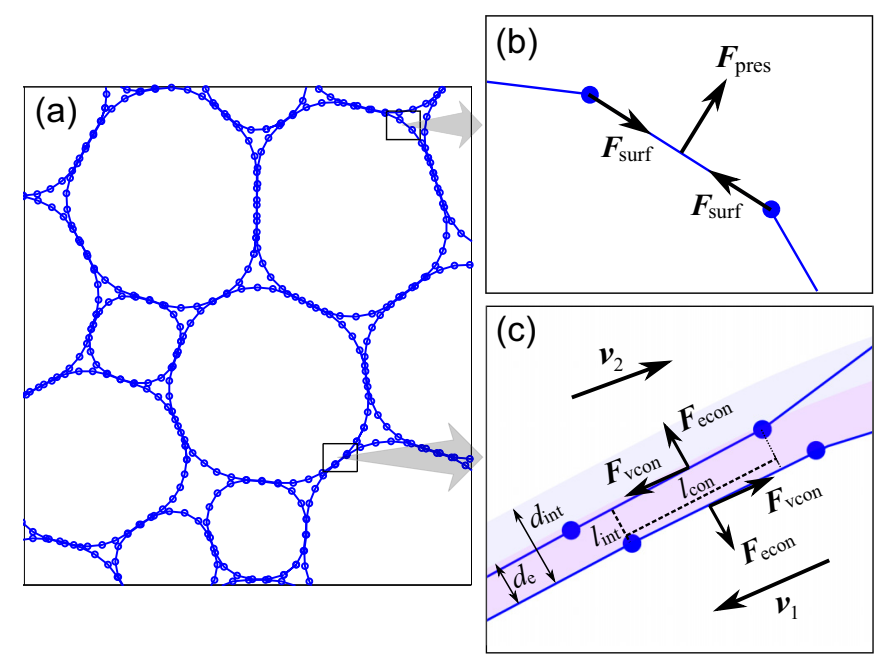

FIG. 1. Panel (a) is a schematic picture of the DySMaL model. Bubbles are modeled as segments and nodes, which I call subparticles, with dynamics resulting from pressure differences, surface tension, and interaction forces between bubbles. Panel (b) illustrates how the pressure and surface tension forces act on a segment. Viscous $\left(\boldsymbol{F}_{\text {vcon }}\right)$ and elastic $\left(\boldsymbol{F}_{\text {econ }}\right)$ contact forces between segments are shown in panel (c). The viscous and elastic contact ranges, $d_{\text {int }}$ and $d_{e}$, respectively, and the shortest distance $l_{\text {int }}$ between the segments and the segment overlap $l_{\text {con }}$ are also marked. Forces are calculated for the segments, but for the time integration the forces are appropriately divided to the subparticles (nodes), which are then propagated.

calculated segmentwise from the equation

$$
\boldsymbol{F}_{\text {surf }}=\sigma \frac{l_{\text {seg }}}{l_{\text {av }}} d_{\mathrm{z}} \hat{\boldsymbol{l}}_{\mathrm{seg}}
$$

in which $\sigma$ is the surface tension, $l_{\mathrm{seg}}$ is the length of the segment, $l_{\mathrm{av}}$ is the average length of the segments of the same surface, $d_{\mathrm{z}}$ is the depth of the third dimension, and $\hat{\boldsymbol{l}}_{\text {seg }}$ is a unit vector parallel to the segment. Also note that the factor $l_{\mathrm{seg}} / l_{\mathrm{av}}$ ensures approximately even segment lengths and is included so as to have a numerically stable surface discretization. The pressure force acting on a segment of a surface is given by

$$
\boldsymbol{F}_{\text {pres }}=\left(p_{\text {in }}-p_{\text {out }}\right) l_{\text {seg }} d_{\mathrm{z}} \hat{\boldsymbol{n}}_{\text {seg }}
$$

where $p_{\text {in }}$ is the internal pressure of the bubble, $p_{\text {out }}$ is the pressure of the carrier fluid at the center of the segment, $l_{\text {seg }}$ is its length, and $\hat{\boldsymbol{n}}_{\text {seg }}$ is its outward unit normal vector. The value for the external pressure $p_{\text {out }}$ was fixed in this work, while the internal pressure $p_{\text {in }}$ is calculated from the ideal gas law.

Interactions between suspended objects are assumed to take place across a thin layer of the carrier fluid. The model employs two types of contact force: viscous and elastic. The viscous contact force is determined from

$$
\boldsymbol{F}_{\text {vcon }}=-\mu_{\text {fluid }} \frac{\boldsymbol{v}_{\text {rel }}}{l_{\text {int }}} l_{\text {con }} d_{\mathrm{z}},
$$

where $\mu$ is the viscosity of the carrier fluid, $l_{\text {con }}$ is the length of the overlap region between the segments in contact, and $l_{\text {int }}$ is the shortest distance between the segments. The basic idea is that the two interacting segments are treated as plates with an overlapping area of $l_{\text {con }} d_{\mathrm{z}}$ separated by a layer of carrier fluid, which mediates a viscous force that is directly proportional to the local shear rate $\boldsymbol{v}_{\text {rel }} / l_{\text {int }}$. Computation of the viscous force is cut off at a distance $d_{\text {int }}$, which is larger than the typical liquid-film thickness between the bubbles. A repulsive contact force is applied in order to prevent 
the suspended objects from penetrating each other. This elastic force is described by

$$
\boldsymbol{F}_{\text {econ }}=-k_{\mathrm{e}} l_{\text {con }} d_{\mathrm{z}}\left(1-l_{\text {int }} / d_{\mathrm{e}}\right) \hat{l}_{\text {int }},
$$

where $k_{\mathrm{e}}$ is the contact modulus and $\hat{\boldsymbol{l}}_{\text {int }}$ is a unit vector toward the closest point in the opposing segment. The underlying assumption is that a (static) film of carrier fluid is between the colliding surfaces; the contact modulus $k_{e}$ could be interpreted to represent the bulk modulus of the carrier fluid. Since here the carrier fluid is assumed to be water (bulk modulus in the GPa range), it can be considered incompressible under the circumstances considered in this work as the maximum pressure is of the order $p_{0}=100 \mathrm{kPa}$. Thus the contact modulus just needs to be large enough to be effectively incompressible.

The subparticles separating the segments are then propagated by explicit time integration in which a segment is considered to have mass $m=\rho d_{e} l_{\text {seg }} d_{z}$, where $\rho$ is the density of the carrier liquid. Parameters $d_{\mathrm{int}}, d_{e}$, and $k_{e}$ in the computation of force and simulation time step, $\Delta t$, have a limited physical relevance, which is not discussed in detail here. I refer the reader to [9] for details, and I use the same values therein, i.e., $k_{\mathrm{e}}=10^{5} \mathrm{~Pa}, d_{\mathrm{e}}=10 \mu \mathrm{m}, d_{\text {int }}=20 \mu \mathrm{m}$, and $\Delta t=1 \mu \mathrm{s}$.

\section{RESULTS AND ANALYSIS}

\section{A. Simulation setup}

In this work, the modeled foam consisted of 240 bubbles with a mean radius of $1 \mathrm{~mm}$ and their circumference was discretized into 40 segments. The bubble radii were uniformly distributed $5 \%$ around the mean, which makes the foam quite close to being monodisperse. The bubbles were constrained in a rectangular area $17.25 \mathrm{~mm}$ wide and $47 \mathrm{~mm}$ long with periodic boundary conditions in the longer dimension. The subsequent packing fraction was thus 0.93 . The depth of the pseudodimension $d_{z}$ was set at $2 \mathrm{~mm}$. Base values for other physical parameters are listed in Table I. The system was then subjected to shear by moving the constraining walls at a set velocity. The shearing was started with a wall shear rate of $1161 / \mathrm{s}$, which was then decrementally lowered down to $1.161 / \mathrm{s}$ and each decrement consisted of $2 \mathrm{~s}$ of shearing with a fixed shear rate. It should be noted that the wall shear rate will differ from the foam shear rate, discussed in the next paragraph, as slip is allowed at the walls. Six such runs were made, which I have numbered with Roman numerals from I to VI. The dynamic viscosity and the surface tension of the system were varied between these runs, and the magnitudes of the parameters for each run are displayed in Table II in relation to the base values given in Table I.

For every imposed shear rate decrement, the time-averaged velocity profile of the foam was measured, and a linear fit was then made in order to determine the foam shear rate. The shear stress was measured directly from the viscous contact forces affecting the walls. The resulting shear stress versus shear rate curves are presented in Fig. 2(a). The general shapes of the shear stress versus shear rate curves are similar across all six runs. Curves for runs III $(\square)$ and IV $(\diamond)$ are essentially the same, as the only difference between these runs was the initial bubble configuration. Other runs differed also with regard to the values of viscosity and surface tension, thus creating the differences between the shear stress versus shear rate curves. However, the shapes of the curves suggest that they differ

TABLE I. Base values for physical parameters used in this work.

\begin{tabular}{lccc}
\hline \hline Parameter & Symbol & Value & Unit \\
\hline Fluid density & $\rho_{0}$ & 1000 & $\mathrm{~kg} / \mathrm{m}^{3}$ \\
Dynamic viscosity & $\mu_{0}$ & 1.8 & $\mathrm{mPas}$ \\
Surface tension & $\sigma_{0}$ & 14 & $\mathrm{mN} / \mathrm{m}$ \\
External pressure & $p_{0}$ & 103 & $\mathrm{kPa}$ \\
Mean bubble diameter & $\langle d\rangle$ & 2000 & $\mu \mathrm{m}$ \\
\hline \hline
\end{tabular}




\section{T. KÄHÄRÄ}
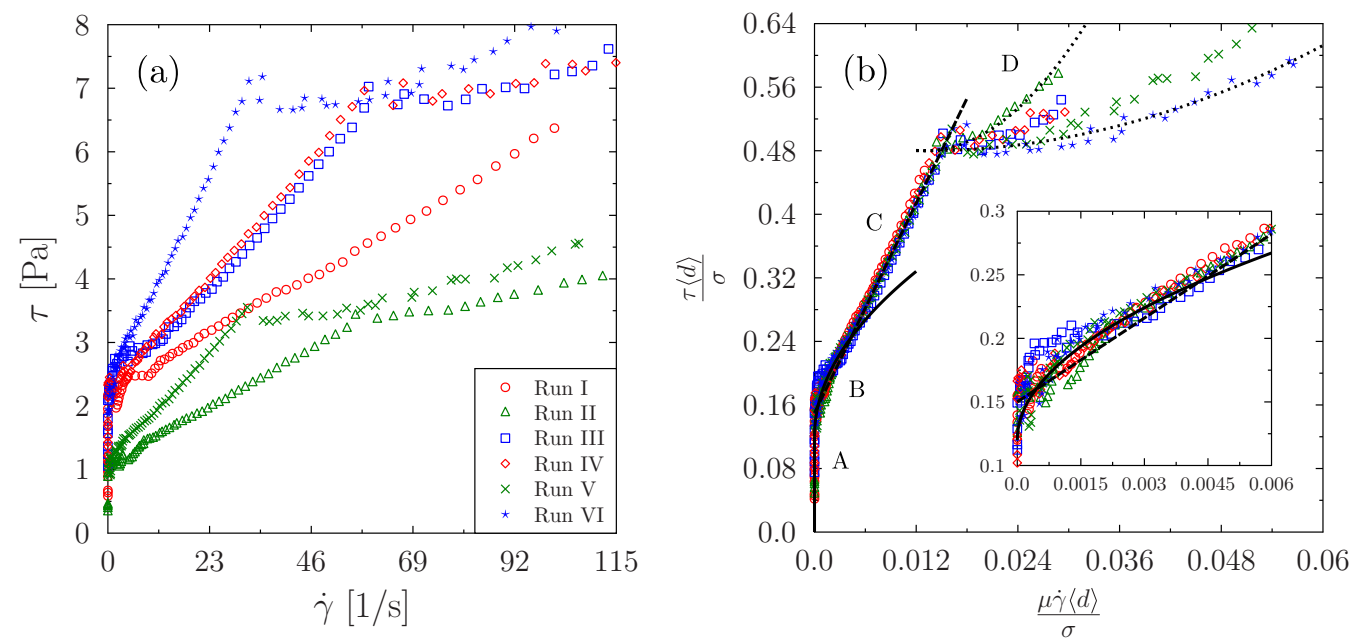

FIG. 2. Panel (a) shows the shear stress vs shear rate profiles for runs I through VI. The run parameters are listed in Table II. Panel (b) has the same data as panel (a), but the axes are scaled to dimensionless units. The curves in panel (b) were added to guide the eye. They and the accompanying letters A, B, C, and D are discussed in the text, while the inset is a magnification of the main figure.

only by a linear scaling of the axes. In Fig. 2(b), I thus plotted the same data using dimensionless quantities on the axes, namely the dimensionless shear stress $\tau\langle d\rangle / \sigma$ and the dimensionless shear rate $\mu \dot{\gamma}\langle d\rangle / \sigma$, also known as the Capillary number. This image shows that the data from the different runs align quite well onto a single curve up to a certain shear rate. I also propose that there can be seen four flow regions with distinct characteristics, labeled A, B, C, and D for the purposes of making references to them easier, and I have included these labels in figures where appropriate.

\section{B. Rheological response to shear}

Starting from larger shear stresses there is first region D, which looks like it could be characterized by a shear thickening power-law constitutive equation, but it is unclear whether this behavior persists at even higher shear rates. Also in this region, the dimensionless scaling seems not to align the data, indicating that the relative importance of the physical properties of the system is not fully captured by the capillary number. When the dimensionless shear stress decreases to a value $\tau\langle d\rangle / \sigma \approx 0.5$, an abrupt transition to region $\mathrm{C}$ is encountered with a significant change in foam viscosity $(d \tau / d \dot{\gamma})$ happening right at the transition. Also below this transition, the relation between shear stress and shear rate becomes linear, characterizing region $\mathrm{C}$, and the overall behavior in this region is subsequently

TABLE II. Physical parameters for different runs relative to the base parameters listed in Table I. The runs are numbered using Roman numerals, and the symbols correspond to the symbols used in Figs. 2(a), 2(b), and 5.

\begin{tabular}{lccc}
\hline \hline Run & Symbol & $\mu / \mu_{0}$ & $\sigma / \sigma_{0}$ \\
\hline I & $\circ$ & 1 & 2 \\
II & $\triangle$ & 1 & 1 \\
III & $\square$ & 2 & 2 \\
IV & $\diamond$ & 2 & 2 \\
V & $\times$ & 2 & 1 \\
VI & $\star$ & 4 & 2 \\
\hline \hline
\end{tabular}




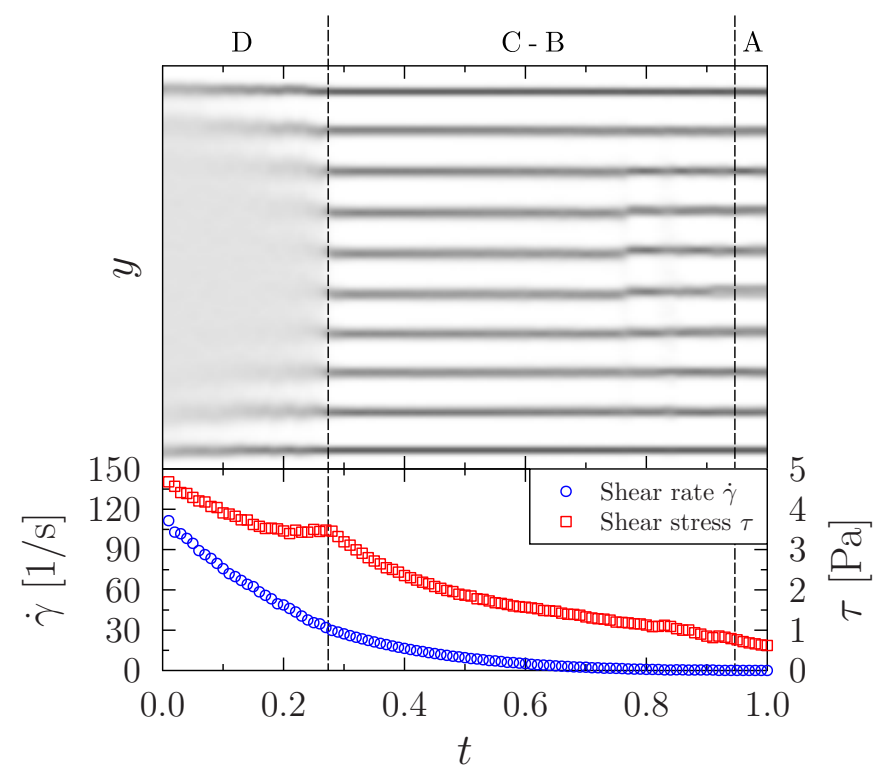

FIG. 3. The shear rate, shear stress, and bubble location probability distribution for run $\mathrm{V}$ are displayed as a function of time given in arbitrary units. In the upper part of the figure, the probability of finding a bubble centroid at a given $y$ coordinate (shearing happens in the $x$ direction) is shown with a darker shade, meaning a higher probability. In the lower part are the corresponding shear rates and shear stresses.

similar to that of a Bingham plastic. As the shear stress is decreased further, the system transitions smoothly to region $\mathrm{B}$, in which there is a shear thinning power-law relation between the shear stress and shear rate. Finally, a region is reached at the lowest shear stresses where the foam shear rate goes to zero, indicating a presence of a yield stress.

Combined regions A and B seem to be consistent with the usual Herschel-Bulkley power-law constitutive equation often used for foams, usually with a power-law exponent somewhere around $1 / 2$ [2]. However, here I make no attempt to assign a value to the exponent since due to the additional degrees of freedom brought by the transition to region $\mathrm{C}$ the value would be highly dependent on how I treat this transition in the fitting procedure. Together, regions A, B, and C can be understood in terms of models such as those in Refs. [10-12], in which the material transitions from an elastic behavior A to a quasistatic phase B, where the system has time to relax after each plastic deformation, finally arriving at a dynamic flowing regime $\mathrm{C}$, where the plastic deformation rate is greater than the relaxation time. Region $\mathrm{D}$, on the other hand, does not fit into an established theoretical framework for foams. Qualitatively similar behavior has been observed in experiments with microemulsions and surfactant micelles, e.g., [13-15], but not to my knowledge in foams. The likely answer to this discrepancy can be inferred from [16], in which the data show that there is a critical shear stress above which bubbles are broken during steady shear, and this critical shear stress is quite close to the values reached in the simulation. Since the simulation will not allow bubbles to break, the rheological response should significantly differ from actual foam when the critical shear stress is reached. Thus my conclusion is that region $\mathrm{D}$ is a result of the model dynamics and emerges beyond the applicable range of the model regarding foams.

Sample curves are included in Fig. 2(b) to illustrate the different behavior in the different regions. The formula $\tau=a+b \dot{\gamma}^{n}$ is used with $n=1 / 2,1$, and 2 in regions $\mathrm{B}, \mathrm{C}$, and $\mathrm{D}$, respectively. The exponents are chosen (not fitted) to highlight the general trends in the regions; thinning, linear, and thickening and best-fit values could vary significantly depending on the fit procedure as there are extra degrees of freedom in choosing the transition points from one exponent to the other. In region $\mathrm{D}$, I have included two curves to represent the extreme cases. 


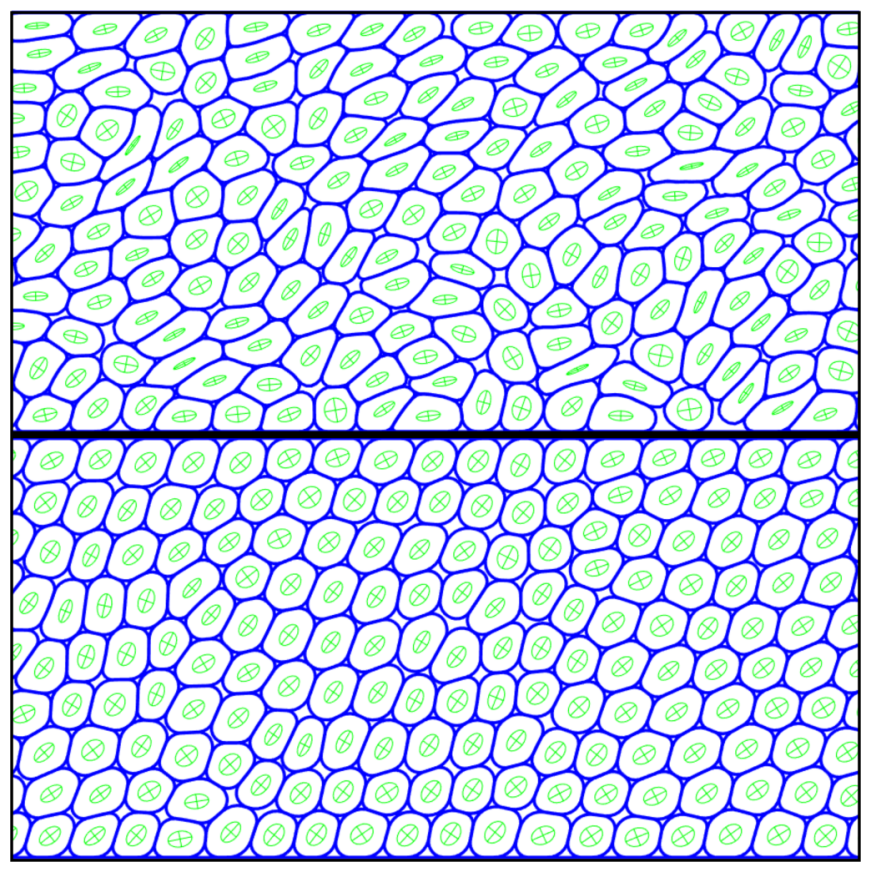

FIG. 4. Two snapshots of the simulated foam with fitted ellipses superimposed. The upper snapshot corresponds to flow region D while the lower one corresponds to region C. Both snapshots are from run V.

\section{Foam structure and bubble deformation}

Next I investigate foam structure and bubble arrangement during shear. The probability of finding a bubble centroid at a certain $y$ coordinate (the system is sheared in the $x$ direction) at different shear rates and corresponding stresses is shown in Fig. 3. From this it is evident that the flow is laminar, in the sense that the bubbles are restricted to distinct layers, in regions $\mathrm{B}$ and $\mathrm{C}$, whereas in region D the flow is erratic and the layer structure is lost. In [17] it was shown that the laminar flow structure appears also for polydisperse foams at sufficiently high shear rates, but at lower shear rates polydisperse foam bubbles have collective swirly trajectories that are not seen here. The absence of the swirly trajectories might be caused by the monodisperse nature of the foam (only $5 \%$ diameter variation), which causes the foam to crystallize at lower shear rates, and it assumes a structure close to the normal hexagonal structure of perfectly monodisperse foam; experimental results such as those in [18] support this idea to some degree. Similar simulations of polydisperse foam should be made with the DySMaL model in order to verify if this explanation is correct or if there is a real discrepancy present. According to [19], an ordered foam is still described by the Herschel-Bulkley power-law constitutive equation at small shear rates but with a power-law exponent of $2 / 3$. This would still be consistent with Fig. 2(b), where an exponent $1 / 2$ is shown, because due to the considerable spread in the data at low shear rates and the uncertainty as to where to transition from region $\mathrm{B}$ to $\mathrm{C}$, enough freedom would be given to quite easily make a reasonable fit with an exponent of $2 / 3$. However, due to the aforementioned reasons, an unbiased fit would be very difficult to do given the data, and thus at this point I will not even attempt to assign a definite value to the exponent.

To explain the structure loss at the transition between regions C and D, I look at bubble deformation, which is characterized by the ratio of semiminor and semimajor axes of an ellipse fitted to the surface profile of a bubble. These are illustrated in Fig. 4. The average bubble deformation is shown in Fig. 5(a), region C, as a function of the dimensionless shear stress. In region D the bubbles are highly deformed, thus this region stands out from the data. This explains both the loss of structure (as the deformed bubbles are unable to form ordered layers) and the different rheological behavior 

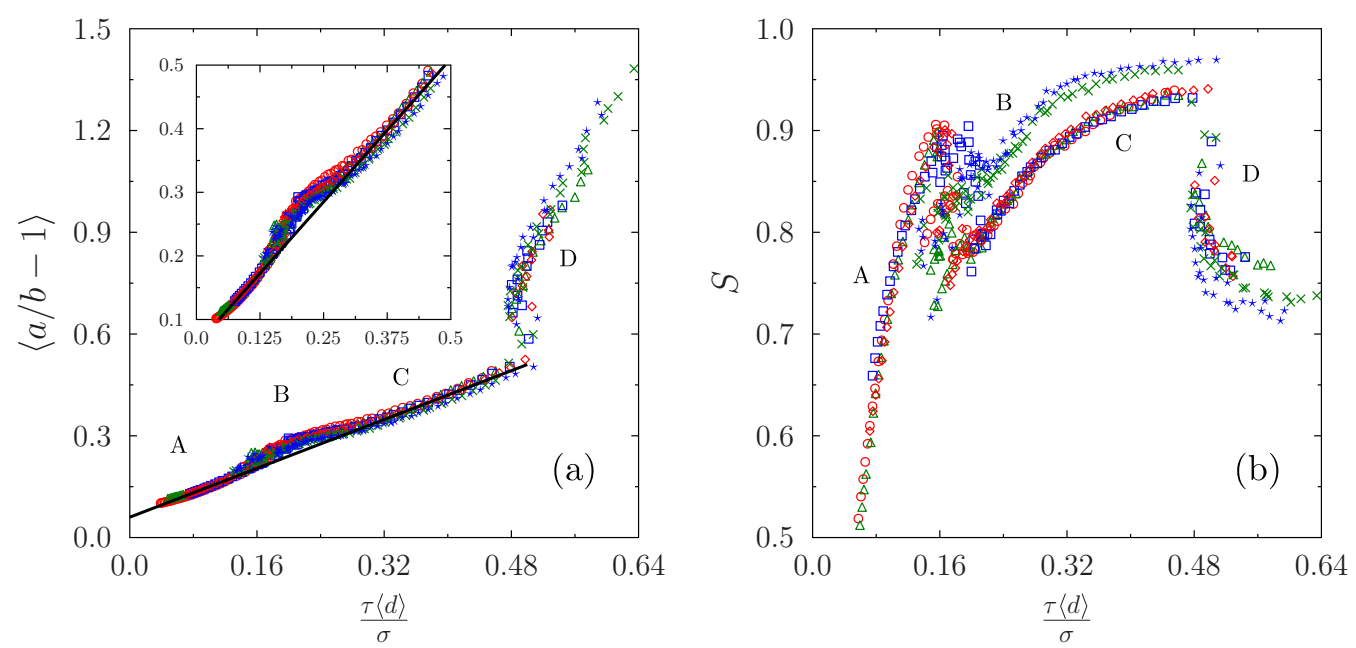

FIG. 5. Panel (a) shows the average deformation of bubbles in runs I through VI, as characterized by the ratio of semimajor $a$ and semiminor $b$ axes of an elliptical fit to the bubble, as a function of the dimensionless shear stress $\tau\langle d\rangle / \sigma$. The inset in panel (a) is a magnifications of the main figure. Panel (b) shows the nematic order parameter (discussed in the text) as a function of the dimensionless shear stress $\tau\langle d\rangle / \sigma$. The accompanying letters $\mathrm{A}, \mathrm{B}, \mathrm{C}$, and D in both panels mark the different flow regimes and are discussed in the text.

from actual foams, which would break under such large deformation due to capillary instabilities. In regions $\mathrm{A}, \mathrm{B}$, and $\mathrm{C}$, the bubble deformations are more modest and the ratio of the semiaxes seems to grow linearly with the shear stress. This is illustrated with a line placed on the data in Fig. 5(a), region $\mathrm{C}$. However, a deviation from the linear behavior can be seen that is located at a stress interval corresponding roughly to region $\mathrm{B}$. It should also be noted that the average deformation increases with shear stress also below the yield stress (i.e., region A), and the foam behaves similarly to an elastic material in this region. Lastly, I investigate the orientation of bubble deformations partly motivated by the importance of nematic order in the rheology of micellar solutions $[13,15]$. The orientation is quantified by the (two-dimensional) nematic order parameter, $S=2\left\langle\cos ^{2}(\theta)\right\rangle-1$, in which $\theta$ is the angle between the individual and system average bubble orientation given by a fitted ellipse. This nematic order parameter is shown in Fig. 5(b), region C, as a function of the dimensionless shear stress $\tau\langle d\rangle / \sigma$. In flow regions $\mathrm{A}, \mathrm{B}$, and $\mathrm{C}$, the order generally decreases with decreasing stress, while abrupt changes in the order happen at transitions between regions $\mathrm{A}$ and $\mathrm{B}$ and regions $\mathrm{C}$ and $\mathrm{D}$. The system is most ordered at the point of transition between regions $\mathrm{C}$ and $\mathrm{D}$. In region $\mathrm{D}$, the order quickly increases with decreasing stress when approaching the transition to region $\mathrm{C}$ starting from a seemingly constant value of approximately 0.75 at the highest stresses. From all of this I conclude that bubble deformations and their orientation are strongly associated with the rheological responses in the system. This is true particularly in region $\mathrm{D}$, although bubble breakup would likely happen in actual foams prior to or at the transition point from region $\mathrm{C}$ to $\mathrm{D}$. The abruptness of the transition and its precise connection to the bubble breakup process and the associated critical shear stress are possible avenues for further study.

\section{SUMMARY}

In this paper, I simulated linear shearing of foam using the DySMaL model. I varied the carrier fluid viscosity and the surface tension of bubbles, and I found that the results scale with those parameters. The shear stress versus shear rate data lay on a single curve when presented in terms of the dimensionless shear stress and shear rate. I also found that at lower shear rates, encompassing regions $\mathrm{A}, \mathrm{B}$, and $\mathrm{C}$, the behavior of the model is consistent with soft glassy rheology models, such 


\section{T. KÄHÄRÄ}

as those in Refs. [10-12], and disk model simulations [17,20]. However, at higher shear rates, the simulation produces an unexpected rheological response labeled region D that is not explained by, nor seen in, the above-mentioned models. Data from [16] show that the transition to region D is quite close to the critical shear stress at which foam bubbles break, which is not allowed by the model. Other shortcomings of the model, besides not allowing for bubbles to break, is that the carrier fluid is not explicitly modeled, and as a consequence the model does not take properly into account certain details of lubricated interactions such as dynamic adhesive and repulsive forces between interfaces or viscous interactions over extended distances. These are all effects that arguably become more relevant the higher the shear rate. Also, as the simulated system is small, finite-size effects may play a role. Thus it is reasonable to conclude that the model does not accurately describe foams beyond the transition point to region D, particularly as I am not aware of experimental results for foams that would produce directly comparable rheological behavior, although the rheology of foams has been studied extensively even at high shear rates and stresses [2]. However, the presence of region D is certainly an interesting and real consequence of the model dynamics and worth further study in its own right, but especially when analogous behavior can be seen in certain microemulsions and surfactant systems [13-15] with quite different microscopic origins. Thus the model can be potentially useful even at higher shear rates if the studied system better matches the model assumptions, i.e., the material consists of deforming bubbles (or grains, etc., for which capillary instabilities do not occur) that are significantly more resilient against rupture, or if the system geometry or composition does not favor bubble breakage.

\section{ACKNOWLEDGMENTS}

I thank M. Kataja and K. Mattila for comments on the manuscript. This work was financially supported by the Academy of Finland, Projects No. 264427 and No. 288526. Computational resources were provided by CSC-IT Center for Science.

[1] S. R. Derkach, Rheology of emulsions, Adv. Colloid Interface Sci. 151, 1 (2009).

[2] S. Cohen-Addad and R. Höhler, Rheology of foams and highly concentrated emulsions, Curr. Opin. Colloid Interface Sci. 19, 536 (2014).

[3] D. Weaire and S. Hutzler, The Physics of Foams (Clarendon, Oxford, 1999).

[4] N. Kern, D Weaire, A. Martin, S. Hutzler, and S. J. Cox, Two-dimensional viscous froth model for foam dynamics, Phys. Rev. E 70, 041411 (2004).

[5] D. J. Durian, Foam Mechanics at the Bubble Scale, Phys. Rev. Lett. 75, 4780 (1995).

[6] Q. Sun and S. Hutzler, Lattice gas simulations of two-dimensional liquid foams, Rheol. Acta 43, 567 (2004).

[7] P. Rognon and C. Gay, Soft dynamics simulation. 1. Normal approach of two deformable particles in a viscous fluid and optimal-approach strategy, Eur. Phys. J. E 27, 253 (2008).

[8] P. Rognon and C. Gay, Soft dynamics simulation. 2. Elastic spheres undergoing a $T_{1}$ process in a viscous fluid, Eur. Phys. J. E 30, 291 (2009).

[9] T. Kähärä, T. Tallinen, and J. Timonen, Numerical model for the shear rheology of two-dimensional wet foams with deformable bubbles, Phys. Rev. E 90, 032307 (2014).

[10] P. Sollich, F. Lequeux, P. Hébraud, and M. E. Cates, Rheology of Soft Glassy Materials, Phys. Rev. Lett. 78, 2020 (1997).

[11] P. Marmottant and F. Graner, Plastic and viscous dissipations in foams: Cross-over from low to high shear rates, Soft Matter 9, 9602 (2013).

[12] S. M. Fielding, Shear banding in soft glassy materials, Rep. Prog. Phys. 77, 102601 (2014).

[13] J.-F. Berret, D. C. Roux, G. Porte, and P. Lindner, Shear-induced isotropic-to-nematic phase transition in equilibrium polymers, Europhys. Lett. 25, 521 (1994). 


\section{NUMERICAL STUDY OF TWO-DIMENSIONAL WET FOAM ...}

[14] K. Krishnan, W. R. Burghardt, T. P. Lodge, and F. S. Bates, Transient rheology of a polymeric bicontinuous microemulsion, Langmuir 18, 9676 (2002).

[15] C. Perge, M.-A. Fardin, and S. Manneville, Surfactant micelles: Model systems for flow instabilities of complex fluids, Eur. Phys. J. E 37, 23 (2014).

[16] K. Golemanov, S. Tcholakova, N. D. Denkov, K. P. Ananthapadmanabhan, and A. Lips, Breakup of bubbles and drops in steadily sheared foams and concentrated emulsions, Phys. Rev. E 78, 051405 (2008).

[17] M. B. Sexton, M. E. Mobius, and S. Hutzler, Bubble dynamics and rheology in sheared two-dimensional foams, Soft Matter 7, 11252 (2011).

[18] Y. Wang, K. Krishan, and M. Dennin, Bubble kinematics in a sheared foam, Phys. Rev. E 74, 041405 (2006).

[19] G. Katgert, A. Latka, M. E. Möbius, and M. van Hecke, Flow in linearly sheared two-dimensional foams: From bubble to bulk scale, Phys. Rev. E 79, 066318 (2009).

[20] I. K. Ono, S. Tewari, S. A. Langer, and A. J. Liu, Velocity fluctuations in a steadily sheared model foam, Phys. Rev. E 67, 061503 (2003). 